Instituto Internacional de Investigación y Desarrollo Tecnológico Educativo INDTEC, C.A.

DOI: https://doi.org/10.29394/scientific.issn.2542-2987.2017.0.0.14.256-274

OAI-PMH: http://www.indteca.com/ojs/index.php/Revista Scientific/oai

\title{
Modelo Teórico de Desempeño del Docente Universitario para la Construcción del Conocimiento
}

Autora: Josefa María Ramírez Becerra Universidad Nacional Experimental "Rafael María Baralt", UNERMB josefa chepa@hotmail.com Trujillo, Venezuela

\section{Resumen}

La presente investigación tiene como propósito proponer un modelo teórico de desempeño del docente universitario para la construcción del conocimiento en la Universidad Politécnica Territorial del Estado Trujillo Mario Briceño Iragorry Extensión Trujillo. Para ello está sustenta teóricamente en aportes de autores como Alba, (2011), Aneca, (2009), Avolio, (2009), Day, (2008) entre otros. Metodológicamente, es un diseño de campo, no experimental, transaccional. Los resultados obtenidos permitirán dar respuesta a los objetivos de la investigación por medio de la elaboración de conclusiones concretas. Por esto, de manera concluyente, el docente universitario, debe demostrar competencias en su praxis educativa hacia una pedagogía donde haya la fusión de la práctica y la teoría; la construcción del conocimiento debe aportar información y entrar en consonancia con la nueva producción científica, dentro y fuera del contexto socio-educativo.

Palabras clave: desempeño docente; construcción del conocimiento; educación universitaria. 


\title{
Theoretical Model of Performance of University Teacher for the Construction of Knowledge
}

\begin{abstract}
The present research aims to propose a theoretical model of performance of the university teacher for the construction of knowledge at the Polytechnic University of Trujillo Mario Briceño Iragorry Extension Trujillo. For this, it is theoretically supported by contributions from authors such as Alba, (2011), Aneca, (2009), Avolio, (2009), Day, (2008) among others. Methodologically, it is a field design, not experimental, transactional. The results obtained will allow responding to the objectives of the research through the elaboration of concrete conclusions. For this, conclusively, the university professor must demonstrate competences in his educational praxis towards a pedagogy where there is the fusion of practice and theory; The construction of knowledge must contribute information and be in harmony with the new scientific production, within and outside the socio-educational context.
\end{abstract}

Keywords: teaching performance; knowledge construction; university education.

Date Received: 22-10-2016

Date Acceptance: $19-12-2016$ 


\section{Introducción}

El proceso enseñanza aprendizaje se centra en la transformación de estructuras/conceptos, apoyados en los conocimientos previos de los individuos, dentro del cual es fundamental el protagonismo de los estudiantes en la construcción de significación de la realidad circundante, provocando cambios en los objetos, así como en ellos mismos. En ese sentido, el desempeño del profesor universitario para siglo XXI comporta un cambio epistemológico o de concepto, siendo urgente situar el proceso de aprendizaje del estudiante como eje del nuevo paradigma docente, enfatizando la capacidad de construir conocimiento conjuntamente, profesor-participantes, para sobrevivir con éxito en esta nueva complejidad social.

De allí que, el docente debe ser capaz de desempeñar su trabajo efectiva y eficazmente sin importar las funciones que realice y metas que se le asignen. El proceso educativo continúa siendo su principal función y responsabilidad, sin embargo, este deberá desarrollar las competencias que les permitan gestionar tanto las diversas actividades de enseñanza dentro del aula como otras exigencias administrativas de la institución para tener como resultado un proceso de enseñanza-aprendizaje de calidad que responda a los requerimientos actuales de la sociedad.

Por ende, la educación universitaria vive un proceso de universalización, lo cual significa que los procesos de reforma, la necesidad de generar modelos innovadores, centrados en el estudiante, utilización de tecnologías, fomento de autogestión, formación integral, así como el aprendizaje a lo largo de la vida, no son elementos distintivos de un continente o país, por tanto, tampoco de una universidad, distinguiéndose solamente experiencias de cada centro, docente y discente en esos procesos.

En ese sentido, Alcántara, (2011) señala que los elementos antes mencionados "confieren a las universidades el papel de generadoras y transformadoras de conocimiento" (pág. 14), por tanto, para estar a la altura 
de esa misión, estas casas de estudio requieren realizar seguimientos a las experiencias educativas para comprobar si están dando respuesta a las necesidades, expectativas de la sociedad.

De allí que, afirme el autor antes mencionado, todo proceso educacional tiene una intencionalidad, el cual depende de la concepción de sociedad, así como de ciudadano (a) a formar, sin embargo, la universalización de la educación universitaria ha conducido a las instituciones universitarias a ser más productivas en cantidad y calidad, con los mismos recursos e incluso menos.

Ahora bien, en la Conferencia Mundial de la Educación Superior (UNESCO, 2009: s.p.), se ratifica que la función de la enseñanza superior para abordar los desafíos mundiales necesita: a). renovar su compromiso de mejorar el acceso, calidad, igualdad entre los sexos; b). lograr la sostenibilidad, para satisfacer necesidades de las sociedades del conocimiento y c). el aprendizaje, investigación e innovación se conecten.

Por tanto, se hace necesario resignificar el desempeño del docente de educación universitaria para que pueda adaptarse a las nuevas condiciones socioculturales del siglo XXI, jugando un papel estratégico en los procesos de desarrollo de los países. Al respecto, Gazzola y Didriksson (2008) señalan algunos aspectos relevantes en cuanto a este nivel educativo en Latinoamérica, así como el papel del profesor en él: a). incremento de la investigación científica; b). impacto de nuevas tecnologías; c). desarrollo de nuevas carreras y áreas de conocimiento con base interdisciplinaria, así como la creciente importancia de la internacionalización lo cual hace necesarios nuevos modelos de formación, aprendizaje e innovación (pág. 46).

Cabe destacar que la educación universitaria contemporánea tiene como función principal orientar al estudiante hacia la plenitud de su actualización y expansión, por tanto, es fundamental la presencia de un 
facilitador con conocimientos para guiarlo (a) conscientemente frente a diversas situaciones cotidianas.

Por esa razón, el profesor universitario debe desempeñarse satisfactoriamente, reflexionando ante la realidad cambiante para ofrecer a los estudiantes, las herramientas necesarias para construir sus conocimientos adaptándolos a su realidad posibilitando la transformación de la sociedad mediante la generación de saberes.

De ahí, la importancia del artículo donde se pretende proponer un modelo teórico de desempeño del docente universitario para la construcción del conocimiento en la Universidad Politécnica Territorial del Estado Trujillo Mario Briceño Iragorry Extensión Trujillo, lo cual permitirá examinar los saberes que el estudiante posee, con la finalidad de orientarlo a la construcción de conocimientos individuales pero elaborados en colectivo. Aunado a esto, esta realidad amerita ofrecer a los estudiantes las estrategias que les permitan aprovechar las oportunidades educativas a su alcance.

Desde esa perspectiva, señalan Zabala y Arnau (2011), "es fundamental que el profesor posea una sólida formación disciplinar e intelectual, la cual le permita desarrollar conocimientos, habilidades, actitudes orientados a resolver situaciones inéditas, insuficientemente presentes en los sistemas educativos actuales" (pág. 43). Es decir, esas competencias permiten adaptar el proceso de enseñanza aprendizaje de los estudiantes a sus necesidades, así como a los continuos cambios de la sociedad del conocimiento, transformando el modelo tradicional de enseñanza basado en la adquisición de saberes en uno sustentado en la capacidad de resolver situaciones a lo largo de la vida.

\section{Bases Teóricas}

En este espacio de la investigación, referido a las bases teóricas reseñaré la información de la literatura disponible, tanto del desempeño del 
docente universitario como el de la construcción del conocimiento con ello, se vislumbrará un abordaje del contexto lo que permitirá construir el andamiaje teórico que dará sustento a las intencionalidades del estudio. los cuales se presentan a continuación.

\subsection{Desempeño del Docente Universitario}

La educación universitaria implica un cambio de conciencia del estudiante para involucrarlo en la lucha y transformación social dentro de una orientación consustanciada con los valores planteados en la Constitución de la República Bolivariana de Venezuela, enfocándose, por tanto, en la construcción de conocimientos como una vía para el logro de esa meta, para lo cual se requiere de un profesor humanista, constructivista con enfoque crítico de la realidad.

El profesor universitario es un orientador facilitador del proceso de aprendizaje, contribuyente al desenvolvimiento del espíritu crítico reflexivo del estudiante, por medio del desarrollo de actividades tanto académicas como administrativas. Este ejercicio de la criticidad debe sustentarse en el abandono definitivo de los métodos basados en la memorización y repetición que lo limita en la realización de análisis, discusión, participación entre otros aspectos.

En ese sentido, la dinámica de la profesión académica está profundamente marcada por la naturaleza de las actividades que supone su ejercicio, las cuales son exclusivas del profesor universitario, pues en la construcción de su tipo ideal se conjugan la práctica de la docente, investigación y extensión, específicas de este nivel.

Por tanto, puede decirse que el desempeño docente se entiende como el cumplimiento de sus funciones ejercidas en diferentes campos: el contexto sociocultural, entorno institucional, ambiente del aula, incorporando para ello una triple dimensión: académica, investigación, extensión, las cuales permiten 
al profesor adaptarse a los cambios propuestos en la educación universitaria venezolana.

Dentro de ese contexto, Alba (2011) explica que los profesores universitarios "son los responsables de hacer realidad la transformación de la educación universitaria...donde el estudiante tenga un papel activo en la construcción de su conocimiento, existan actividades didácticas complementarias, acción tutorial próxima a éstos y se utilicen materiales de apoyo" (pág. 13-35).

Se puede inferir entonces, que el desempeño del docente universitario implica cambiar la metodología de enseñanza utilizada tradicionalmente, pasando de aquella transmisora, a una centrada en el estudiante y su proceso de aprendizaje, convirtiéndose en guía de éste, facilitando el logro de competencias a alcanzar por él, para lo cual, el profesor debe crear los contenidos adecuados, además, ser agente motivador de sus educandos en la construcción de sus conocimientos.

\subsubsection{Aspectos del Desempeño del Docente Universitario}

El desempeño del docente universitario se entiende como el conjunto de funciones y roles que hacen posible su acción, ajustado a la demanda del entorno, así como a la normativa educativa vigente, la cual se apoya en una serie de cualidades modeladoras de su comportamiento en términos de involucramiento, compromiso con el trabajo.

Dentro del ámbito educativo, el desempeño es visto como el trabajo realizado por el docente a partir de instrucciones encaminadas al logro de los objetivos, haciendo énfasis en alcanzar la calidad, la excelencia y la eficiencia del proceso académico, para lo cual según, Day, (2008) "debe reunir aspectos como: competencias, conocimiento de las teorías de aprendizaje y responsabilidades profesionales" (pág. 32). 
Con base en lo planteado, puede afirmarse que el desempeño del docente universitario, viene a constituir un campo de acciones orientadas a la actualización disciplinaria, comprensión de su papel como agente dinamizador de las transformaciones sociales, reconocimiento de la práctica como objeto de estudio, permitiéndole estar al tanto de los avances en las disciplinas didácticas; es decir, debe estar concebido desde un enfoque interdisciplinario, para su quehacer educativo cotidiano.

Ahora bien, para poder brindarle un sentido completo al desempeño del profesor universitario, se considera la docencia como una práctica educativa integral, donde éste es un líder capaz de mediar el aprendizaje en sus estudiantes, ser creativo e intelectual, además inspirarlos en búsqueda y adquisición del conocimiento. Partiendo de las consideraciones anteriores, el desempeño del docente es definido por Day (ob.cit) como:

El conjunto de habilidades personales, profesionales y técnicas que demuestran los docentes en el ejercicio de su labor. Se refiere a toda la gama de actividades que un profesor realiza en sus trabajos a diario y que está relacionado con la preparación de clases, el asesoramiento individualizado de los alumnos, el dictado de clases, la calificación de los trabajos asignados, las coordinaciones con otros docentes..., así como la participación en programas de capacitación (pág. 38).

De acuerdo con la definición anterior, el desempeño de los docentes implica no sólo funciones académicas, sino administrativas, por lo cual debe priorizar la planificación de estrategias didácticas y recursos para el aprendizaje, que deben estar íntimamente vinculados con planes, programas de estudio, así como ajustarse a las características de sus educandos. A continuación, se definen los aspectos del desempeño del docente mencionados con anterioridad. 


\subsubsection{Competencias Docentes}

El desarrollo de las competencias depende en gran medida, de una docencia centrada en el aprendizaje, donde a partir del uso de estrategias variadas, se permite al estudiante identificar problemas y presentar soluciones. Al respecto, Perrenoud (2007), afirma que "éstas representan una capacidad para movilizar varios recursos cognitivos para hacer frente a un tipo de situaciones" (pág. 87).

Considerándose para ello tres aspectos: a). Las competencias no son en sí mismas conocimientos, habilidades o actitudes, aunque movilizan, integran, orquestan tales recursos; b). Esta movilización sólo resulta pertinente en situaciones únicas, aunque se la puede tratar por analogía con otras ya conocidas y c). Su ejercicio pasa por operaciones mentales complejas, sostenidas por esquemas de pensamiento, los cuales permiten determinar y realizar una acción relativamente adaptada a la situación.

De acuerdo con la afirmación anterior, las competencias docentes son el resultado de una combinación de varios recursos: actividades o prácticas profesionales a realizar correspondientes a esquemas propios de cada persona; las actuaciones, que constituyen los resultados evaluables de las acciones realizadas.

De acuerdo con lo antes planteado, la competencia docente se puede valorar positivamente si integra simultáneamente la disciplinar (saber), metodológica (hacer), social (estar) aplicándolas de manera coordinada en la práctica. Pero sobre todo, un profesor es competente si sabe transferir esta interrelación de estrategias complejas a nuevas situaciones educativas y de aprendizaje.

En síntesis, concreta la investigadora, las competencias docentes comprenden todas las acciones conducentes a desarrollar la praxis pedagógica, en donde se fusionan teoría y práctica para construir un conocimiento o determinado saber mediante secuencias ordenadas de lo 
simple a lo complejo, así como modelos didácticos que permitan aplicar herramientas didácticas en la consecución de actividades propias de las áreas académicas.

\subsubsection{Conocimiento de las Teorías de Aprendizaje}

La calidad de la educación está íntimamente ligada a la acción o praxis académica, la cual debe ser pensada con base en una reflexión de los cambios sociales, por ello, ésta no debe verse como una superflua relación de los contenidos de una disciplina. En consecuencia, el profesor universitario requiere tener dominio de las teorías de aprendizaje, fundamentalmente aquellas con orientación constructivista, que permitirán trabajar un modelo de enseñanza con una perspectiva diferente. Entre éstas cabe mencionar: cognitivismo, constructivismo, pedagogía social.

Al respecto, el cognitivismo: de acuerdo con Saettler, (2008) se basa principalmente "en las representaciones y proceso mental, considerando al alumno una entidad activa, capaz de construir, resolver problemas" (pág. 17). Además, admite entender cómo el conocimiento es representando en la memoria, reteniendo la información desde las estructuras cognoscitivas, agregándola a la estructura mental.

De acuerdo con lo anterior, la teoría cognitivista se fundamenta en los procesos y la representación del conocimiento, más allá del cambio de conducta observable de un alumno expuesto a un estímulo-respuesta, propuesto por el conductismo, enfatizando en el saber en lugar de la respuesta, viendo al estudiante como activo, constructivo, capaz de resolver problemas. Dentro de ese contexto cabe destacar que el desempeño docente debe basarse en una pedagogía interactivo-constructiva, fundamentada en los siguientes modelos teóricos:

De acuerdo al constructivismo, el aprendizaje significativo; se distingue por dos características, la primera es que su contenido puede relacionarse de 
un modo sustantivo, no arbitrario o al pie de la letra, con los conocimientos previos del estudiante, y la segunda es que éste ha de adoptar una actitud favorable para tal tarea, dotando de significado propio a los contenidos que asimila.

Referente a la pedagogía social, la enseñanza como proceso de mediación social: se basa en que los escenarios sociales pueden propiciar oportunidades para el trabajo cooperativo de los estudiantes, el cual estimula la crítica mutua, los ayuda a perfeccionar su trabajo y permite el apoyo entre ellos.

Puede inferirse entonces que el docente universitario debe poseer dominio no sólo de los contenidos disciplinares sino, ir más allá, al conocer las teorías de aprendizaje para determinar cuáles aportes de éstas son aplicables en su realidad cotidiana, pues no todos sus estudiantes aprenden de la misma manera, requiriendo por tanto, diversas formas de mediación.

\subsubsection{Responsabilidad Profesional}

La responsabilidad profesional implica la conciencia del docente sobre las propias necesidades de aprendizaje, su compromiso y participación en la responsabilidad social de su institución, así como en las políticas nacionales de educación. Entonces, se refiere a aquellas dimensiones del trabajo docente, las cuales van más allá del trabajo de aula involucrando, primeramente, su relación con su profesión, pero también con sus pares, escuela, comunidad, sistema educativo.

De acuerdo con Agencia Nacional de Evaluación de la Calidad y Acreditación de España, (ANECA, 2009) "la responsabilidad profesional del docente en cuanto su principal propósito y compromiso es contribuir a que todos los estudiantes aprendan" (pág. 45). Para ello, reflexiona consciente, sistemáticamente sobre su práctica y la reformula, contribuyendo a garantizar una educación de calidad para todos los estudiantes. 
Desde esa perspectiva, el compromiso del profesor con el aprendizaje de sus estudiantes implica, por una parte, evaluar sus procesos de aprendizaje con el fin de comprenderlos, descubrir sus dificultades, ayudarlos a superarlas y considerar el efecto que ejercen sus propias estrategias de trabajo en los logros de los educandos. Por otra parte, implica formar parte constructiva del entorno donde se trabaja, compartir, aprender de sus colegas y con ellos; sentirse un aprendiz permanente, además de un integrante del sistema educativo. Al respecto, Senlle (2008) señala:

La responsabilidad es una actitud que exige cumplimiento a alguien que no tiene motivación para comprometerlo, se da a las personas que están entrenadas, se sienten preparadas y quieren demostrar que pueden tomar nuevas responsabilidades a su cargo. Con la formación apropiada y permitiendo la participación, hay que darla a los que la piden, previo estudio de cada caso, cuando las cosas se hacen bien no es necesario andar detrás de la gente exigiendo que la cumplan (pág. 65).

La definición anterior, al llevarla al desempeño del docente universitario implica que éste debe reflexionar críticamente sobre las estrategias desarrolladas, sus efectos en los aprendizajes de los estudiantes, evaluando cómo se cumplieron los objetivos propuestos o si éstos se comprometieron con las actividades de aprendizaje. A partir de ese análisis, la responsabilidad profesional del profesor le exige reformular su práctica pedagógica para hacerla más efectiva, de manera de satisfacer las necesidades de aprendizaje de los estudiantes

\subsection{Funciones Básicas del Docente Universitario}

La actividad del docente universitario es compleja pues incorpora elementos personales, disciplinares, pedagógicos, siendo su tarea principal el acto educativo dentro de su área de conocimiento o disciplina científica. Pero, también debe poseer saberes, habilidades, destrezas, valores que deben 
aprender a su vez los futuros profesionales, así como buscar la forma de aprendizaje en los contextos donde tanto profesores como estudiante interactúan y construyen una dinámica particular.

Por ello, Fernández, (2009) explica que hablar de llevar a cabo un buen desempeño profesional del docente universitario, es "saber adaptarse a las nuevas situaciones planteadas por el estudiantado, el currículo, la estructura de las instituciones educativas $y$, también es remitirse al conocimiento de diversas disciplinas entrecruzadas en la práctica" (pág. 76).

En ese sentido, se infiere que la labor docente es lograr la participación activa de los estudiantes en su proceso de enseñanza aprendizaje, siendo su finalidad orientar, guiar, sostener su actividad constructiva proporcionándoles las ayudas educativas necesarias. Al respecto, Romero, (2010) describe "el cometido y funciones esenciales del profesional de la enseñanza, para lo cual deberá tener una serie de conocimientos relacionados con las teorías de la educación, currículo, cultura del profesor: pedagógicos, sociológicos, psicológicos, didácticos" (pág. 81).

\subsection{Construcción del Conocimiento}

Actualmente, la sociedad es testigo de una revisión de los supuestos epistemológicos que han orientado el desarrollo del conocimiento en todos los ámbitos. Al respecto, Pérez, (2010) señala una nueva manera de mirar la realidad, la cual enfatiza la naturaleza socialmente construida del conocimiento, sugiriendo:

El conocimiento no es objetivo ni estable porque forma parte de la propia realidad conocida, porque es una representación subjetiva, es una construcción interesada, condicionada por los flujos de poder, por el intercambio de tensiones e intereses de esa misma realidad. Así pues, la dialéctica entre conocimiento y realidad, entre objeto y sujeto, difumina constantemente los límites entre ambos, confundiéndose en un juego interminable 
de espejos, en una espiral de influjos convergentes y divergentes (pág. 61).

Lo considerado anteriormente proporciona una plataforma para para interpretar la naturaleza del conocimiento, así como a los procesos a través de los cuales los estudiantes construyen el conocimiento. En ese sentido, se entiende la educación como un proceso de ayuda sistemática, planificada, sostenida para el logro de los aprendizajes. Esa ayuda, tiene como objetivo fundamental facilitar al estudiante el acceso a un conjunto de saberes, habilidades, actitudes consideradas relevantes para una actuación profesional satisfactoria, constructiva y crítica ante el contexto social y cultural del cual forma parte. Por ello, Coll (2008) expresan que la ayuda del profesor al proceso de construcción es:

Sólo ayuda, porque el proceso de construcción en sí mismo es individual e interno, y no puede ser sustituido o determinado completamente desde el exterior; pero ayuda necesaria, porque sin ella es poco probable que construcción permita de manera óptima la apropiación, tanto desde el punto de vista del significado como del sentido, de los contenidos culturales que las situaciones educativas tienen como objeto (pág. 35).

De acuerdo con lo antes señalado se infiere que el docente universitario proporciona, mediante su función de mediador, la ayuda necesaria para promover la construcción del conocimiento en sus estudiantes. Por tanto, el profesor debe ofrecerla a sus estudiantes considerando los siguientes aspectos: a). Las ayudas ofrecidas a los estudiantes requieren ser variadas en cantidad, calidad, proporcionando formas y niveles de apoyo diversos; b). Los tipos y niveles de ayuda que se ofrezcan a los estudiantes deben evolucionar y combinar en función de sus actuaciones y c). Las ayudas ofrecidas deben plantear retos que los estudiantes pueden abordar gracias a lo aprendido previamente. 


\subsubsection{Aspectos de la Construcción del Conocimiento}

Aprender en sentido amplio implica concebir a los sujetos con quienes activamente construyen su comprensión basándose en la experiencia y utilizando sus estructuras previas. En ese sentido, Sager y Osguthorpe (2011) expresan que "el aprendizaje es un proceso de construcción social del conocimiento, así como de cambio conceptual intersubjetivo, reflexivocolaborativo sobre la práctica" (pág. 29).

Cabe señalar que el examinar el proceso de construcción de conocimiento desde una perspectiva social, como lo propone la educación universitaria venezolana, implica focalizar la atención en los contextos en los cuales esto ocurre. Estos conocimientos, señala Tardif (2009: 196), "no se construyen y utilizan en función de su potencial de transferencia y de generalización, están enclavados, embutidos, encerrados en una situación de trabajo a la que deben atender".

Ahora bien, la sociedad actual ha redefinido la articulación de las universidades con su entorno, propiciando para ello tres procesos estratégicos: a). Incorporar la producción del conocimiento interdisciplinario; b). Adoptar un paradigma pedagógico centrado en el aprendizaje continuo; c). Desarrollar una capacidad de vinculación externa para la transferencia y difusión de dicho conocimiento.

Desde esa perspectiva, Pérez, (2010) señala que "el conocimiento es todo proceso de construcción de significados situacionalmente condicionado" (pág. 109). Es decir, para ser capaz de entender su valor, afectos, implicaciones, es clave identificar las posiciones y marcos de referencia desde los cuales los estudiantes elaboran sus interpretaciones, análisis, así como juicios de valor.

Se infiere entonces que la construcción del conocimiento implica una relación dialógica entre teoría y práctica. En opinión de la investigadora, la construcción del conocimiento es un proceso sociocultural que obedece al 
carácter de integralidad del desarrollo de la capacidad transformadora humana, generado en la dinámica de las relaciones entre los sujetos en la sociedad, a través de una constante, sistemática relación, capaz de potenciar su comportamiento en el ser, hacer, saber, convivir.

\section{Metodología}

Metodológicamente la naturaleza de la investigación, se arma en una investigación de campo, de carácter descriptivo, donde la investigación de campo, según Hernández, Fernández y Baptista (2003), consiste en "la aplicación sistemática de procedimientos de la investigación social con el propósito de valorar la conceptualización, el diseño, la aplicación y la utilidad de los programas de acción social" (pág. 35), o sea su finalidad es recoger los datos en el sitio donde se realiza la investigación.

En otras palabras, ésta radica en la observación directa, actuación de personas y situaciones en que ocurren ciertos hechos, por este motivo, la naturaleza de las fuentes determina la forma de obtener los datos. Es decir, se pretende establecer un proceso de investigación en forma directa donde se encuentra la situación problemática objeto de estudio, en este caso en específico en la Universidad Politécnica Territorial del Estado Trujillo Mario Briceño Iragorry Extensión Trujillo, en cuanto se refiere a proponer un modelo teórico de desempeño del docente universitario para la construcción del conocimiento

\section{Conclusiones}

En esta fase del trabajo, se pueden crear algunas conclusiones a las que se ha llegado, luego de la consulta de las fuentes bibliográficas pertinentes al tema con el intento de obtener una visión más profunda de la temática tratada y aportar razones para que el desempeño del docente universitario para la construcción del conocimiento 
El docente universitario, debe mejorar los aspectos referidos a la forma de manejar estrategias en cuanto a abandonar definitivamente los viejos métodos apoyados en la memorización y repetición que sin duda, limita al estudiante en la realización de análisis, discusiones, ser participativo, además de ser crítico y reflexivo de sus propios aprendizajes.

En cuanto, a las competencias docentes su praxis educativa debe dirigirse hacia una pedagogía donde haya la fusión de la práctica y la teoría encaminado a la construcción del conocimiento donde inserte modelos de teorías de aprendizaje que consientan aplicar herramientas en la consecución de actividades propias donde se ejemplifique la responsabilidad profesional.

La construcción del conocimiento en investigación, tiene diferentes alcances e impedimentos importantes en la sociedad, ya que aporta información y entra en consonancia con la nueva producción científica, dentro y fuera del contexto socio-educativo hacia escenarios comprensibles y creativos, ante las corrientes del pensamiento, las competencias académicas, entre otras.

Concluyendo, la construcción del conocimiento es un proceso dialéctico en tanto la fusión de la teoría con la práctica y sociocultural porque obedece al carácter integral en el desarrollo de la capacidad humana, fruto de las recomendaciones entre los sujetos en la sociedad, capaces de potenciar su comportamiento y así centrar la atención en los contextos en los que esto ocurre.

\section{Referencias}

Alba, E. (2011). "Praxis del Docente Universitario". Editorial Magisterio. Bogotá.

Alcántara (2011). "Retos de la Educación Superior para el Siglo XXI". Ediciones OEl. México. 
ANECA (2009). “Desarrollo y Evaluación de Competencias Pedagógicas”. Madrid.

UNESCO (2009). Conferencia Mundial de la Educación Superior. Ediciones UNESCO. París.

Day, C. (2008). "Formar Docentes: Cómo, Cuándo y en Qué Condiciones Aprende el Profesorado". Editorial Narcea. Madrid.

Fernández, M. (2009). "Desarrollo Profesional Docente". Grupo Universitario. Granada, España.

Gazzola y Didriksson (2008). "Verdades y Mitos de la Educación Superior en Latinoamérica”. Ediciones UNESCO. Buenos Aires.

Hernández, Fernández y Baptista (2003). Metodología de la Investigación. McGraw-Hill. México.

Pérez, A. (2010). "La Formación del Profesorado". Editorial Morata. Madrid. Perrenoud, P. (2007). "Diez Nuevas Competencias para Enseñar”. Editorial Grao. Barcelona, España

Saettler, F. (2008). "Psicología del Aprendizaje”. Editorial Pialp. Madrid.

Sager, M. y Osguthorpe, R. (2011). "Educación Docente". Editorial Homosapiens. Buenos Aires.

Senlle, P. (2008). "Gestión de Calidad y Competitividad de las Organizaciones". Editorial Irvin. México.

Zabala, A. y Arnau, L. (2011). "Cómo Aprender y Enseñar Competencias". Editorial Colofón-Graó. México. 


\section{Josefa María Ramírez Becerra \\ e-mail: josefa chepa@hotmail.com}

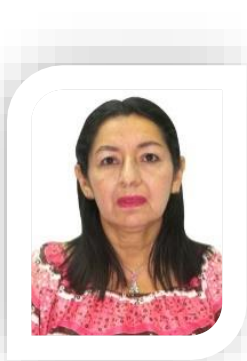

Nacida en Valera, estado Trujillo, Venezuela. Actualmente cursa estudios de Doctorado en Ciencias de la Educación en la Universidad Nacional Experimental "Rafael María Baralt". Especialista en Gerencia de Empresas, cursado en la Universidad Valle del Momboy; Diplomado de Componente Docente en la UNEFA. Actualmente, docente contratada tiempo completo del Instituto Universitario de Tecnología del Estado Trujillo Extensión Trujillo. Desde 13-032010 hasta la presente fecha (6 años) Tutora de Proyectos Socio Integrador y jurado evaluador de los Proyectos Socio Integradores. Delegada de la Unidad de vinculación Socio-Comunitaria. Delegada de Trabajo Social Comunitario.

El contenido de este manuscrito se difunde bajo una Licencia de Creative Commons ReconocimientoNoComercial-Compartirlgual 4.0 Internacional 